\title{
TWO CASES OF SARCOIDOSIS IN ONE FAMILY WITH REVIEW
}

\author{
AHMEDUL KABIR ${ }^{1}$, JAYANTA BANIK ${ }^{2}$, TUSHAR KANTI BARMAN ${ }^{3}$
}

\begin{abstract}
Sarcoidosis is a multisystemic disease that has an immunological basis. We report a family in which two members have been affected with sarcoidosis. The radiological findings of all cases were presented, together with histopathological diagnosis. The particular interest in this report is the fact that two family members we present here are blood relatives.
\end{abstract}

\section{Introduction:}

Sarcoidosis represents a multisystem granulomatous disease the etiology of which is unknown. It mainly involves the lungs, lymph nodes, skin, eyes, bones and the parotid glands. The clinical picture is attributed to the presence of granulomas, which are composed of epithelioid and multinucleated cells with little or no necrosis. ${ }^{1}$ The first ever case of sarcoidosis was probably described by Boeck in 1899, familial sarcoidosis was not described separately until 1923. ${ }^{2}$ There are several case reports of familial sarcoidosis and here we present two blood related sisters in one family who are presented in, Medicine Out Patient Department of Dhaka Medical College and Hospital. There is some evidence of familial clustering and genetic factors are undoubtedly important. Previous case reports of familial sarcoidosis show findings of HLA B8 in association with bilateral hilar lymphadenopathy and spontaneous resolution of disease and an association between HLA A2 and pulmonary changes in the presentation of sarcoidosis is also proposed. The published cases of familial sarcoidosis shows that, it is more common in monozygotic than in dizygotic twins, in like sex pairs than in unlike sex pairs, and in mother-child relations than in father-child relations. ${ }^{2}$ The course and prognosis of the disease depends on the mode of onset of the disease. An acute onset is usually selflimited and a spontaneous resolution is likely, but an insidious onset may leads to relentless progressive fibrosis. ${ }^{3}$

\section{Case 1}

The 30 year old previously fit unmarried woman, presented with non productive cough, recurrent attack of multiple joint pain and fever for 3 months. The cough was intractable and doses not respond to treatment with several antibiotics, bronchodialators and cough suppressant drugs. The joint pain involves the both elbow and knee joint associated with swelling of knee joints, morning stiffness and some restriction of activity. The condition resolves after taking NSAID. The fever was low grade, irregular, mostly comes at night and relives spontaneously without sweating. Her bowel and bladder habit was normal.

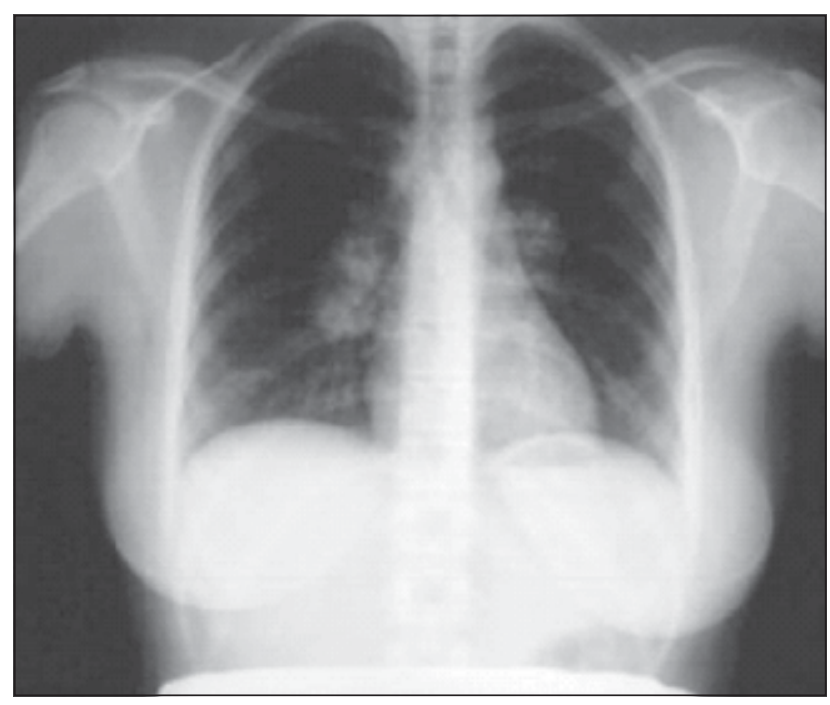

Fig. 1. Chest X-ray of case 1 showing bilateral hilar lympadenopathy

1. Resident Physician (RP), Out Patient, Department (Medicine), Dhaka Medical College Hospital, Dhaka

2. Post-graduate Trainee, Dept. of Medicine, Dhaka Medical College Hospital, Dhaka

3. Medical Officer, Out Patient Department (Medicine), Dhaka Medical College Hospital, Dhaka 


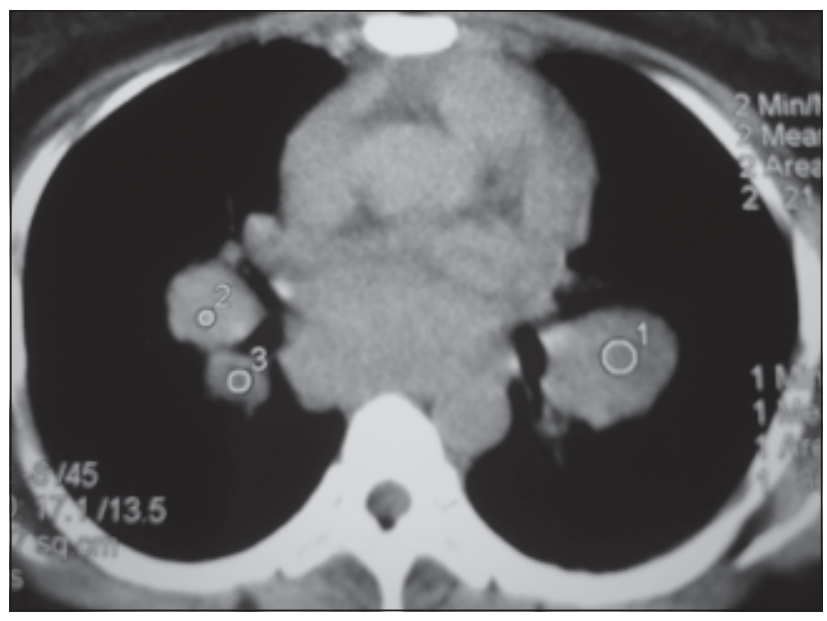

Fig. 2: CT scan of Chest of case 1 showing bilateral hilar lympadenopathy

Her physical examination revealed an alert and oriented lady. Her vitals were the following: pulse, 80/minute and regular; blood pressure, 110/70 $\mathrm{mmHg}$; respiratory rate 14 breaths/minute; temperature, $99^{\circ} \mathrm{F}$. The head and neck examination was normal. The abdomen was soft, non-distended and non-tender with normal liver and spleen span. The neurological, cardiovascular, and chest examinations were unremarkable.

Her complete blood count and Blood film revels, WBC - 9.5 X 10x9/L with a PMN predominance of $69 \%$, Hematocrit - 29\%, Platelets - $300 \times 10 \times 9 / L$, ESR 15,Hb - $10.5 \mathrm{~g} / \mathrm{dl}$ ( Normal: $11.5-16 \mathrm{~g} / \mathrm{dl}$ ), PBF : RBC - Microcytic and hypochromic red cells with moderate anisopoikilocytosis and few pencil teardrop \& target cells , WBC-Mature, Plateletes - Normal. Urinalysis is negative for protein or red cells but Leucocyte count is $10-12 / \mathrm{HPF}$. LFT shows, S.Bilirubin $-1.2 \mathrm{mg} / \mathrm{dl}$, ALT(SGPT) - 22 U/L. A chest radiograph was obtained and showed bilateral hilar lymphadenopathy ( Fig-1). Mantoux test was $00 \mathrm{~mm}$ and sputum ( 3 samples ) for AFB are negative. Then a CT guided FNA was approached from right hilar mass ( Fig-2) and the hitopathological smear shows moderate cellular material containing lymphocytes, polymorphs, pulmonary Macrophage, a few gaint cells \& focal collection of epithelioid cells with a comment of noncaseating granulomatous inflammation compatible with Sarcoidosis.

\section{Case 2}

The second case, 32 years old women, sister of case 1 presented with nonproductive cough, progressive dyspnoea, and occasional wheeze for 2 months. She also complaints about recurrent swelling of legs which resolves spontaneously. Physical examination was unremarkable, but a chest radiograph showed gross bilateral hilar nodular shadowing ( Fig-3). A computed tomographic (CT) scan confirmed the mediastinal and bilateral hilar lymphadenopathy ( Fig-4 ). Her complete blood count, Liver and Renal function test was normal. Mantoux test is $0 \mathrm{~mm}$ and Serum Calcium level is normal. Thyroid function test reveals biochemically euthyroid. A CT guided FNAC from mediastinal mass showing noncaseating granulomatous inflammation compatible with Sarcoidosis.

\section{Discussion:}

Sarcoidosis is an idiopathic multisystem disease most commonly affecting young adults. Most patients present with sarcoidosis between the ages of 20 and 40 , but the disease can occur in children and in the elderly. ${ }^{4}$ Several hundred kindred groups with familial sarcoidosis have been described, and the disease has been observed in twins, more commonly in monozygotic than in dizygotic pairs. There have also been several instances of husband-wife pairs identified, and geographic foci of sarcoidosis among unrelated individuals living closely within a community, arguing for some environmental factors, in the pathogenesis of the disease. Unlike many diseases in which the lung is involved, sarcoidosis favors nonsmokers. ${ }^{4}$

The cause of sarcoidosis is unknown. Various infectious and noninfectious agents have been

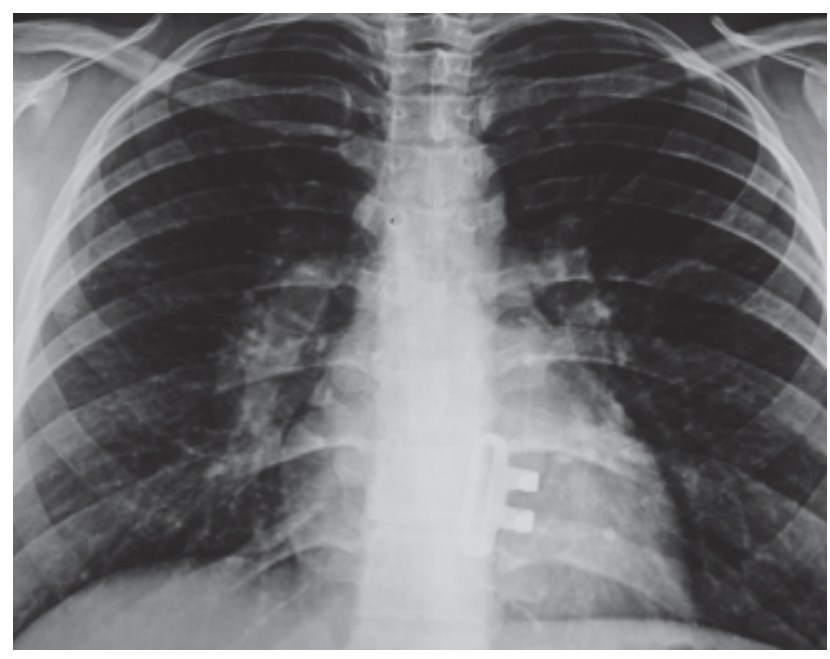

Fig. 3: Chest $X$-ray of case 2 showing bilateral hilar lympadenopathy 


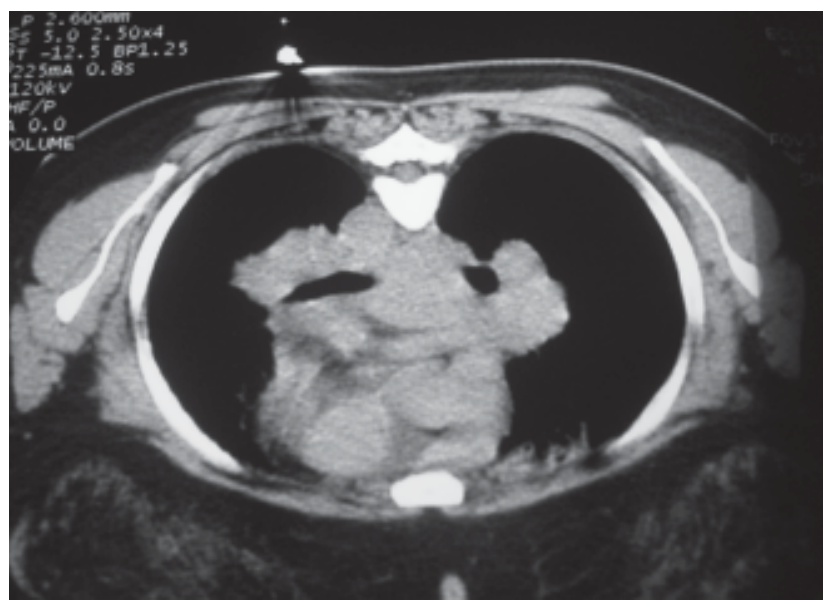

Fig. 4: CT scan of Chest of case 2 showing bilateral hilar lympadenopathy

implicated, but there is no proof that any specific agent is responsible. The current hypotheses of the cause of sarcoidosis, not mutually exclusive, include the following: (1) The disease is caused by a class of persistent antigens, nonself or self, that trigger only the TH1 cell arm of the immune response; (2) the disease results from an inadequate suppressor arm of the immune response, such that $\mathrm{TH} 1$ cell processes cannot be shut down in a normal fashion; or (3) the disease results from inherited (and/or acquired) differences in immune response genes, such that the response to a variety of antigens is an exaggerated, TH1 cell process. Although the disease is believed to result from exaggerated cellular immune responses to a limited class of antigens, no clear patterns in any HLA locus have emerged. ${ }^{4}$ Recently, by using molecular biologic techniques, mycobacteria have been identified in sarcoidal tissues, raising the possibility of $M$. tuberculosis or a related organism as a causative agent. In recent years several pathogenic models suggest that the disease might result from exposure of genetically predisposed individuals to a kind of specific environmental infectious agent of which several DNA viruses ( Epstein-Barr-virus, human herpes virus, retrovirus, HIV) are suspected. ${ }^{5}$

Sarcoidosis is a systemic disease, and thus the clinical manifestations may be generalized or focused on one or more organs. ${ }^{4}$ The clinical manifestations depend on the organ involved. Despite extensive tissue involvement, initially the symptoms may be relatively mild. Progression of the disease in most cases are usually slow with relapses and remissions and eventual healing. Thus, the clinical picture is extremely variable and mainly is the effects of fibrosis and/or pressure exerted by the enlarged structures to the organ involved. ${ }^{1}$

The acute or subacute sarcoidosis develops abruptly over a period of a few weeks and represents 20 to $40 \%$ of all cases. Two syndromes have been identified in the acute group. Lo"fgren's syndrome, includes the complex of erythema nodosum and x-ray findings of bilateral hilar adenopathy, often accompanied by joint symptoms, including arthritis at the ankles, knees, wrists, or elbows. The Heerfordt-Waldenstro" $m$ syndrome describes individuals with fever, parotid enlargement, anterior uveitis, and facial nerve palsy. The insidious form of sarcoidosis develops over months and is usually associated with respiratory complaints without constitutional symptoms. ${ }^{4}$

It commonly affects the lungs causing cough, dyspnea and wheezing with weight loss. ${ }^{1}$ Skin lesions manifest as The most common lesions are erythema nodosum, plaques, maculopapular eruptions, subcutaneous nodules, and lupus pernio. Erythema nodosum, comprising bilateral, tender red nodules on the anterior surface of the legs. The maculopapular eruptions occur on the face around the eyes and nose, on the back, and on the extremities. The lesions on the tip of the nose cause a bulbous appearance (lupus pernio), sometimes associated with varicosities. ${ }^{4}$ Ulcerative skin lesions are rare. ${ }^{6}$

Ocular involvement is commonly manifested as uveitis. Decrease in visual acuity, blurring, irritation with photophobia and excessive lacrimation may also occur. Iritis is followed by peripheral anterior synechia, retinal perivasculitis and spotty retinochoroidal exudates. All these manifestations require early referral for slit lamp examination. ${ }^{1,4}$

Joint involvement mostly involves the large joints, with boggy effusion and thickening of the joint membranes. acute, self-limited polyarthritis may be observed in up to $40 \%$ of sarcoid patients, other manifestations, such as chronic arthritis $(1-4 \%)$ or symptomatic muscle involvement $(<0.5 \%)$ are much less common. ${ }^{3}$ Bone Sarcoidosis presented as back pain and spondylolisthesis is very rare. ${ }^{7}$

Other systems involved include renal, which may be presented as FSGS (Focal Segmental Glomerulosclerosis ) that may progressed to end stage renal failure, Glomerulonephritis is rare (except membranous nephropathy). sarcoidosis and FSGS can 
only be suggested in the presence of idiopathic nephrotic syndrome. ${ }^{8}$

Cardiac sarcoid may be isolated, or associated with systemic involvement, the interventricular septum base is commonly affected, which leads to heart block or arrhythmia and sudden death. ${ }^{9}$

All components of the nervous system can be involved in sarcoidosis. common manifestations of neurosarcoid include optic nerve dysfunction, papilloedema, palate dysfunction, hearing abnormalities, hypothalamic and pituitary abnormalities, chronic meningitis, and, occasionally, space-occupying lesions. ${ }^{4}$

Parotid enlargement is a classic feature of sarcoidosis, Sarcoid can present as an isolated pancreatic lesion mimicking pancreatic carcinoma. ${ }^{4}$ Upper respiratory tract, testicles and various other organs are also involved. ${ }^{1}$

On Laboratory investigation, common abnormalities in the blood include lymphocytopenia, an occasional mild eosinophilia, an increased erythrocyte sedimentation rate, hyperglobulinemia, and an elevated level of angiotensin-converting enzyme (ACE). False-positive tests for rheumatoid factor or antinuclear antibodies can be observed. Hypercalcemia is rare. Other serum abnormalities relate to involvement of specific organs such as liver, kidney, or endocrine glands. Because the lung is involved so commonly, the routine chest film is almost always abnormal. The three classic $\mathrm{x}$-ray patterns of pulmonary sarcoidosis are type I-bilateral hilar adenopathy with no parenchymal abnormalities; type II-bilateral hilar adenopathy with diffuse parenchymal changes; and type III-diffuse parenchymal changes without hilar adenopathy. The type III pattern is sometimes split into two categories, with films that show fibrosis and upper lobe retraction classified separately. Computed tomography of the chest is rarely helpful for either diagnosis or prognosis but can identify early fibrosis. A "ground-glass" appearance is often associated with an active alveolitis but more likely results from the granulomas. The lung function abnormalities of sarcoidosis are typical for interstitial lung disease and include decreased lung volumes and diffusing capacity with a normal or supernormal ratio of the forced expiratory volume in 1 second to the forced vital capacity. The gallium-67 lung scan is usually abnormal, showing a pattern of diffuse uptake. ${ }^{4}$
Bronchoalveolar lavage typically demonstrates an increased proportion of lymphocytes, most of which are members of the activated TH1 subset of CD4+T lymphocytes. The remaining cells are mostly alveolar macrophages. In patients with significant fibrosis, a few neutrophils are also found. Eosinophils are rare. ${ }^{4}$

The other laboratory features of sarcoidosis depend on the specific organ involved. The MRI features of sarcoid in long bones are not specific, and must be differentiated from metastatic disease, multiple myeloma, lymphoma, osseous hemangioma, and disseminated granulomatous infection. ${ }^{3}$

However, the diagnosis of sarcoidosis mainly depends on tissue studies with biopsies taken from lymph nodes, conjunctivae, scalene fat pad, skin lesions, buccal mucosa or lung tissue. ${ }^{1}$

There are many reports of HLA associations in familial sarcoidosis. The association of HLA B8 is established previously and recently a new association of HLA A2 has also been proposed. The data that was published previously also suggest that HLA DR3 confers a good prognosis and HLA DR5 leading to increased susceptibility for the development of the disease. $^{2}$ Of the two genetically related members in our report, we are unable to do HLA typing due to technical problem, but this investigation may add further information, either in favour or against of these hypotheses.

The treatment of sarcoidosis is mainly supportive and corticosteroids is the cornerstone of treatment. Prednisone is given as $1-2 \mathrm{mg} / \mathrm{kg} /$ day until symptoms improve then tapered to reduce the dose to $15 \mathrm{mg}$ every other day for a total period of six months. Sequential oral and inhaled corticosteroid therapy may be an alternative treatment regimen for sarcoidosis. ${ }^{1}$ Methotrexate is usually the second-line medication. Drugs that are used in refractory cases, including indomethacin, oxyphenbutazone, chloroquine, hydroxychloroquine, thalidomide, infliximab, etanercept, pentoxifylline, tacrolimus, $p$ aminobenzoate, allopurinol, levamisole, azothioprine, and cyclophosphamide; Cyclosporine is ineffective for the pulmonary manifestations of the disease; anecdotal reports suggest that it may be useful in extrathoracic sarcoid not responding to glucocorticoids. ${ }^{4}$ Diet low in calcium was advised.

We treated the patients with Prednisolone and they respond well with the treatment. 


\section{Conclusion:}

Sarcoidosis is a chronic multiorgan disease. For a typical case, the diagnosis of sarcoidosis is made by a combination of clinical, radiographic, and histologic findings. In a young adult with constitutional complaints, respiratory symptoms, erythema nodosum, blurred vision, and bilateral hilar adenopathy, the diagnosis is almost always sarcoidosis. Because sarcoidosis can occur in almost any place in the body, like tuberculosis or syphilis, it can be confused with many other disorders.So the differential diagnosis of sarcoidosis must cover a wide range including neoplastic diseases such as lymphoma or disorders characterized by a mononuclear cell granulomatous inflammatory process, such as the mycobacterial and fungal disorders. No blood findings are diagnostic of the disease. The chest $\mathrm{x}$-ray cannot be used as the sole criterion for the diagnosis of sarcoidosis. While the finding of bilateral hilar adenopathy is the hallmark of this disease, a similar pattern is occasionally observed in lymphoma, tuberculosis, coccidioidomycosis, brucellosis, and bronchogenic carcinoma. The histologic evidence is mandatory for a definitive diagnosis of sarcoidosis and overall prognosis in sarcoidosis is good. The present case reports may be helpful in drawing attention to the occurrence of sarcoidosis in family members as early recognition and proper management may prevent complications such as blindness, pulmonary insufficiency, and renal impairment. Our report also raises the possibility of an association between HLA and familial sarcoidosis and it will be interesting to see whether further reports support this or not.

\section{References:}

1. Al-Momen J, AlSanae HM. Childhood Sarcoidosis Case Reports. Kuwait Medical Journal 2007; 39 (1): 56-58

2. Elford J, Fitch P, Kaminski E, McGavin C, Wells I P. Five cases of sarcoidosis in one family: a new immunological link?, Thorax 2000; 55: 343-344.

3. Chaudhry S, Michael L. Imaging Findings in Sarcoid of the Humerus, Richardson: Radiology Case Reports. 2006;1:154-158.

4. Kasper, Fauci, Longo, Braunwald, Hauser, Jameson. Harrison's Principles of Internal Medicine, $16^{\text {th }}$ edition, page 2017-2023

5. Tamme T, Leibur E, Kulla A. Stomatologija, Sarcoidosis (Heerfordt syndrome): A case report. Baltic Dental and Maxillofacial Journal, 2007; 9:6164.

6. Albertini JG, Tyler W, Miller OF. Ulcerative sarcoidosis. Case report and review of the literature. 1997; 133.

7. Morgan SS, Aslam MB, Mukkanna KS, Ampat G. A rare presentation of sarcoidosis, back pain and spondylolisthesis. Journal of Bone and Joint Surgery 90: $240-42$.

8. Francisco JV, Veronese. Pulmonary sarcoidosis and focal segmental glomerulosclerosis: case report and renal transplant follow-up. Nephrol Dial Transplant 1998; 13: 493-495

9. Veinot JP, Johnston B. Cardiac sarcoidosis-an occult cause of sudden death: a case report and literature review. 1998; 43. 